\title{
Using Context Information for Tailoring Multimedia Services to User's Resources
}

\author{
José M. Oliveira ${ }^{1}$ and Eurico M. Carrapatoso ${ }^{2}$ \\ ${ }^{1}$ INESC Porto, Faculdade de Economia, Universidade do Porto, \\ ${ }^{2}$ INESC Porto, Faculdade de Engenharia, Universidade do Porto, \\ rua Dr. Roberto Frias, 378, 4200-465 Porto, Portugal \\ $\{$ jmo, emc $\}$ @inescporto.pt
}

\begin{abstract}
The integration of multiple network access technologies in the terminal is one of the most important trends in $4 \mathrm{G}$ networks. This integration places telecommunications service providers, and in particular operators, under the significant challenge of being able to transform their services in order to adapt them to a great variety of delivery contexts. This paper describes a generic methodology for the adaptation of telecommunications services provided in the context of an operator. A number of scenarios are examined which show how Parlay middleware can be used by trusted service providers to easily access network resources and context information for enhancing location-based and adaptable multimedia telecommunications services.
\end{abstract}

Keywords: Seamless Service Provisioning, Parlay Middleware, Context Gathering, Multimedia Adaptation.

\section{Introduction}

In the recent years, telecommunications have evolved to a new and highly competitive environment, especially for communications service providers. Factors such as the convergence between fixed and mobile networks, between broadcast and communications, and between mobile and Internet led to the need of seamless service provision across different types of networks and totally independent of terminal technologies. This independence requirement gains particular importance in the provision of next generation services, characterized not only by location based features, but also by context-aware features, multimedia contents and user mobility. The acceptability of new services will only be effective if the user has the possibility to access them anywhere, in any technological circumstances, even in roaming scenarios. This user requirement places multimedia service providers under the significant challenge of being able to transform their services in order to adapt them to a great variety of delivery contexts.

This paper presents a generic adaptation methodology suitable to adapt telecommunications services to different access mechanisms and connectivity capabilities. The adaptation methodology, introduced in the following section, is based mainly on two fundamental constructions: the Multimedia Presentation

D. Krishnaswamy, T. Pfeifer, and D. Raz (Eds.): MMNS 2007, LNCS 4787, pp. 138 148, 2007.

(C) IFIP International Federation for Information Processing 2007 
Model (MModel) and media adapters. The former enables a device independent specification of the user interface, including the modelization of time-based features. Media adapters basically enable the materialization of the user interface specification in the most suitable format for a particular user context.

The integration of context information in telecommunications services and the development of context-sensitive applications have grown enormously in the present decade, mainly due to the increase mobility of users and to the research activities on ubiquitous and pervasive computing. We follow the approach of directly accessing user context information managed by network operators, using the open middleware offered by Parlay. Parlay APIs and Web services are designed to enable the creation of telecommunications applications outside the traditional network space and business model. Network operators consider Parlay a promising architecture to stimulate the development of Web service applications by third party providers, which may not necessarily be experts in telecommunications. This approach is currently promoted in the telecommunications field, namely by the 3GPP IP Multimedia Subsystem (IMS) standardization effort 11.

To explore the advantages of the adaptation methodology to produce multimedia services adapted to the user context, the paper presents in Section 3 a media adapter targeted to the SMIL language. In Section 4 we evaluate the proposed adaptation methodology using a case study service. We detail in several scenarios how this service uses the Parlay middleware to easily access network resources and information. In Section 5 we compare our approach for the dynamic adaptation of multimedia services with some related work. The paper finalizes reporting the main conclusions in Section 6.

\section{A Methodology for the Adaptation of Telecommunications Services}

The adaptation methodology presented in this section is based on the MModel, an abstract construction that holds an XML description of the user interface, including the complete list of available user interactions [2]. The objective is to create a mediator (the Adaptation System), between the service and the user, to manage all user interactions with the service. According to the actual service session parameters, the conversion of the MModel to a specific format and the adaptation of service contents to the right media type is achieved through specific implementations of the media adapter entity.

Fig. 1 places the Adaptation System into the IMS layer architecture. The Adaptation System and the Parlay Gateway, both positioned in the middleware layer, help applications, seen in this context as telecommunications services, to abstract from the below network infrastructure, which can involve completely different access technologies, such as WLAN, UMTS or circuit-switched networks. During the service adaptation process, the Adaptation System interacts with the Parlay Gateway, taking advantage of the functionalities offered by the Parlay APIs and Web services, as will be described later in Section 4 


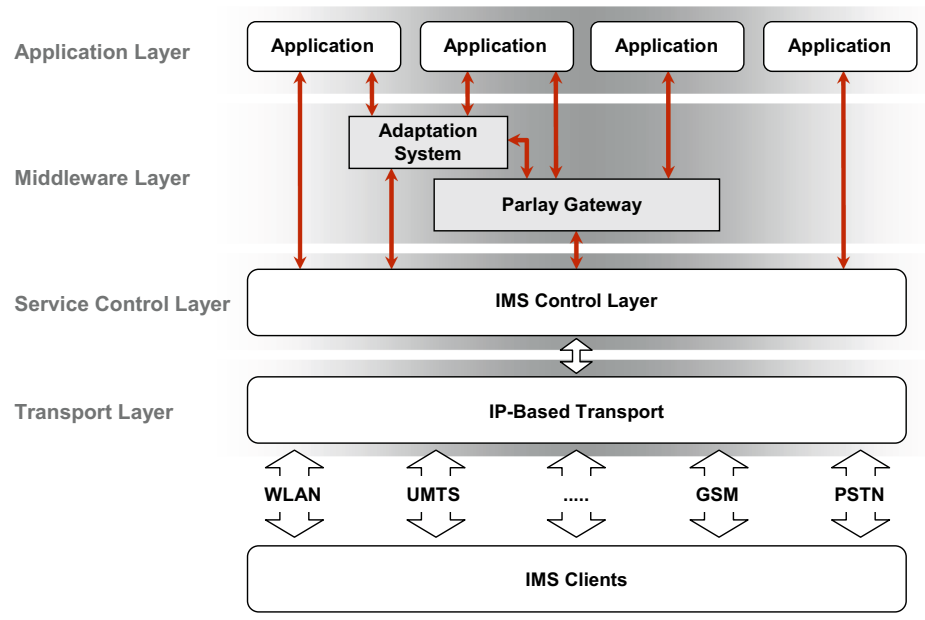

Fig. 1. IMS layer architecture

Each Adaptation Session is composed by two objects: the MModel, which can be seen as the representation of a service on the adaptation system side; and the Interpreter, which can be seen as a mediator between the Media Adapter and the Service Listener.

The user, after accessing his service provider, interacts with the portal application and chooses to use a service that he has previously subscribed. This interaction is transmitted to the Portal Listener, which triggers the creation of a new service session manager on the adaptation system side. This service session manager communicates with the service provider that offers the chosen service so that a service session manager on the service provider side and the associated listener can be also created (see Fig. 2).

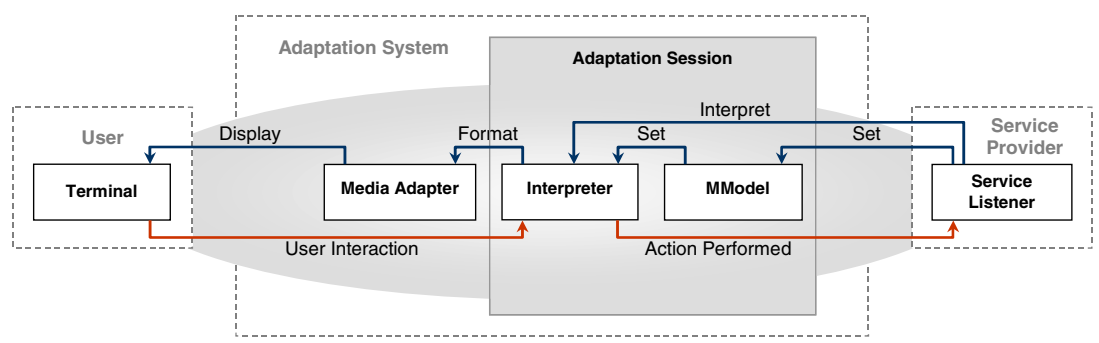

Fig. 2. Service adaptation cycle

The Service Listener is responsible for filling the MModel object with the user interface specification. This specification is usually divided into several parts, corresponding to the several views of the user interface. With the MModel set on 
the adaptation system, the service adaptation process starts, following a cycle approach (see Fig. 2). In each step, the adaptation is only carried out over one of the MModel parts. For that, the Service Listener sets the focus on the MModel part that should be adapted before it requests the Interpreter to interpret this part.

When the user receives a new interface in the terminal device, he has the opportunity to interact with it, leading the service to evolve to a new state. Each user interaction is characterized by the possible inputs introduced by the user and the order to go to a new state, typically represented by the reference of the interaction element. The Interpreter is the object that communicates the interaction description to the Service Listener. Using a parser, the Service Listener decodes the interaction and routes it to the right method of the service logic, which will process it and trigger the appropriate internal actions to answer the user interaction. Independently of the service, these changes in the service state will lead to an update of the MModel object, integrating the changes in the service state. The Service Listener sends a new user interface specification to the MModel and set the focus on the MModel part that it wants to be active.

\section{The SMIL Adapter}

This section presents a media adapter prototype specifically designed to enable the introduction of multimedia contents and to support time-based features in telecommunications services 3. The objective was to specify and develop a media adapter that should produce a final form user interface from an input interface specification defined using the MModel format. In what concerns the output format, the media adapter should produce information in an interactive multimedia format, targeted to the Web. We chose SMIL, a language defined by the World Wide Web consortium (W3C), which enables the specification of multimedia presentations for delivery over the Web 4. The choice of SMIL had the additional advantage of allow an easy mapping with the MModel due to its XML-based structure.

The adaptation methodology proposed by us prescribes the definition of the user interface structure as the initial point of the adaptation process, corresponding to the instantiation of the MModel object. Then, the interface structure is maintained stable during the different methodology phases, having no influence on the dynamic change of the user interface during the service session lifetime. External factors, such as the device characteristics, the network bandwidth conditions or the user available time, have a much more active role in the user interface dynamic generation. Since in mobile computing, the user context information is very dynamic by nature, the capability to dynamically detect this information at the beginning of each adaptation cycle is decisive to achieve acceptable results with the adaptation methodology.

\subsection{Gathering the User Context}

The SMIL Media Adapter follows the approach of using the Parlay middleware to obtain user context related information directly from the network [5]. The Parlay 
Application Programming Interfaces (APIs) are a standardization effort carried out by the Parlay Group since 1998. These APIs enable application developers to access telecom network capabilities through an open interface. The final objective is to open up the network resources to trusted parties, such as service developers, content providers and service aggregators. The Parlay Group started in 2000 an update of their APIs to become Web services, with the intention of migrating to the all-IP convergence approach started at that time.

The appropriate Parlay APIs and Web services for obtaining the desired context information are: the Terminal Capabilities API, wich enables an application to synchronously retrieve the terminal capabilities of a specific terminal, as a CC/PP profile [6] the Terminal Status and Location Web Services, which provide terminal status and location services, allowing applications to obtain the geographical location and the status (e.g., reachable or busy) of fixed, mobile and IP based telephony users; the Connectivity Manager API, which allows applications to constantly and efficiently obtain QoS monitoring information concerning the network conditions of user connections to service providers (restricted to an enterprise scale); and the Presence Web Service, which offers a synchronous and an asynchronous approach for obtaining presence and availability information.

\subsection{SMIL Generation Process}

The SMIL Media Adapter uses the XSL Transformations (XSLT) 7] of the Extensible Stylesheet Language (XSL), which is a language specified by the W3C consortium for defining XML documents transformations and presentations. XSLT allows the specification, in a standardized way, of functional transformations from one XML tree to another, which are specified in a document usually known as a style sheet. The SMIL Media Adapter defines one such style sheet, which establishes the transformation rules from the MModel XML tree to the SMIL XML tree. The use of a relatively simple approach for transforming the MModel specification into a concrete delivery format using a XSLT transformation is driven by the guarantee that the calculations for the visual layout and timing of the media items produces an interface MModel tree that is known to meet the current user context, as will be detailed later in this section.

Currently, most of the time-based formats are presentation formats. The SMIL was built as a Web presentation language, which supports hyperlinks to different parts of the presentation or to any external destination URI [8]. A drawback of the SMIL language is the lack of support for input processing (usually designated as forms processing in the Web world). The SMIL linking elements are not a suitable choice to manage user interactions over a SMIL interface, since they are interpreted directly by the SMIL player, at the client side, being completely hidden from the service provider, which is not desirable in a service provision context. The intrinsic lack of support for forms processing should not be seen as a real drawback of the SMIL language, since currently the focus of W3C specifications is to separate the forms processing from specific languages, creating a standardizing way of treating them. This standardization effort is materialized into the XForms standard [9], which may be considered as an autonomous 
module that contains forms semantic-related XML elements and attributes and should be integrated in different XML based languages, such as SMIL, extending these languages.

The SMIL Media Adapter internally uses the constraints theory to establish dependencies between user interface variables according to the user context information gathered. The main idea behind the constraint-based approach for the automatic generation of multimedia presentations is to use a constraint solver system to determine one (preferably the best) solution for a set of variables that are interrelated by constraints.

The constraint solver system chosen to be used in the context of the SMIL Media Adapter was the $\mathrm{ECL}^{\mathrm{i}} \mathrm{PS}^{\mathrm{e}}$ constraint logic programming system [10, which not only offers a Java interface, but also the possibility to define application dependent constraints, which is a very useful feature for multimedia applications, where the relations between the different interveners cannot be easily specified using typical numerical domains constraints. In addiction, ECL ${ }^{\mathrm{i}} \mathrm{PS} \mathrm{e}^{\mathrm{e}}$ supports the backtracking and unification features of logic programming, combining them with the domain reduction properties of constraint programming, resulting in what is usually called a Constraint Logic Programming (CLP) system. The use of the backtracking mechanism gives a dynamic characteristic to the specification of constraints. Alternative constraints can be specified when an initial constraint causes a failure, preventing the application from crashing or not providing any information.

The communication between the ECL ${ }^{\mathrm{i}} \mathrm{PS}^{\mathrm{e}}$ system and the SMIL Media Adapter is made through a queue mechanism provided by the ECL ${ }^{\mathrm{i} P S}$ /Java interface (see Fig. 3). Two information queues are defined (ToEclipseQueue and FromEclipseQueue), which allow the SMIL Media Adapter to respectively send to and retrieve information from the ECL ${ }^{\mathrm{i}} \mathrm{PS}^{\mathrm{e}}$.

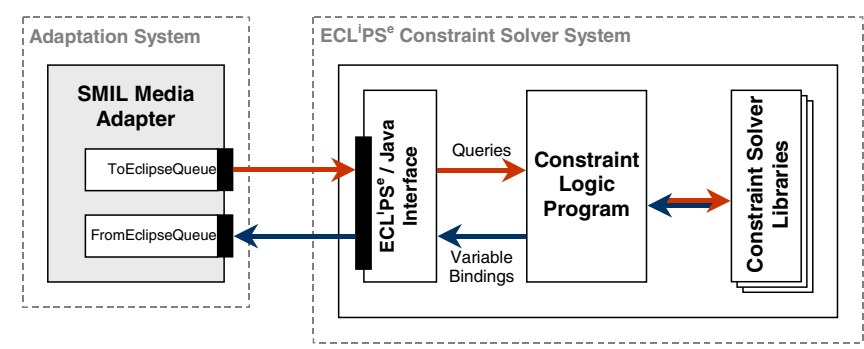

Fig. 3. Communication with the constraints solver system

When the internal ECL ${ }^{\mathrm{i}} \mathrm{PS}$ C Constraint Solver Libraries find a solution for the constraint problem, the SMIL Media Adapter extracts it and updates the MModel focused part, namely with the spatial and temporal values found for each media item. 


\section{Parlay Middleware Usage Scenarios}

As a proof of the concept we have implemented a prototype service with the objective of evaluating the most relevant research aspects proposed in this paper. An overview of the Customer Care service in presented in Fig. 4, which clearly shows one of the characteristics that is addressed by this service: the service ubiquitous access in terms of terminals and networks.

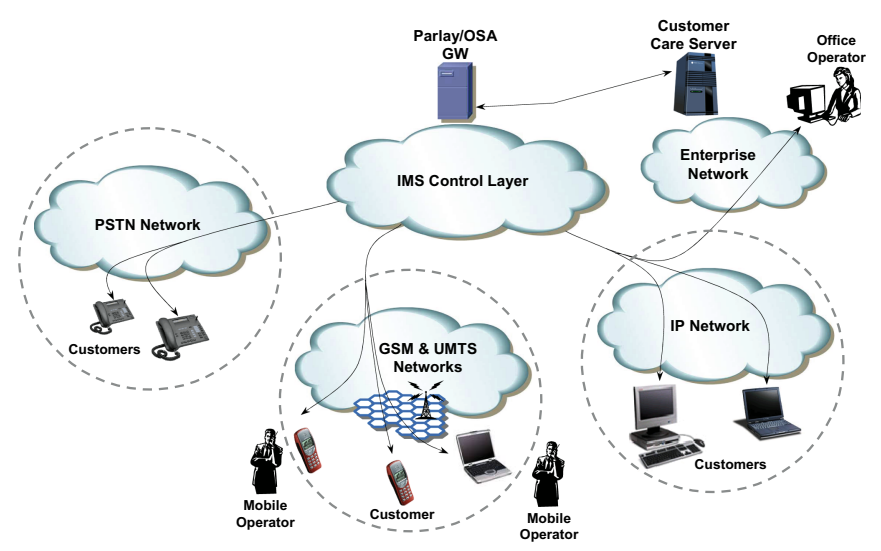

Fig. 4. Overview of the Customer Care service

The Customer Care service suitability for evaluating the use of telecommunications middleware in the service adaptation to the user context is directly related with the two features provided by the service: interactive tutorials, where the user interactively accesses information about products and services of a given supplier, organized as tutorials; and online help, where the user requests the establishment of an audio or videoconference with one of the available online operators. The multimedia flavor of the interactive tutorials enables the demonstration of the media adaptation to different terminals and networks used to access the service. The multi-party characteristic of the online help feature enables the demonstration of network transparency and service adaptation to location information, through the use of Parlay middleware.

\subsection{Call Establishment Scenario}

This scenario happens when a user requests online help from the Customer Care service. Initially, the service uses the Parlay Terminal Status Web service to determine the reachable online operators (see Fig. (5) . Then, using the Terminal Location Web service, the Customer Care service determines the nearest available operator and send him an SMS, using the SMS Web service, notifying him of the user request. 


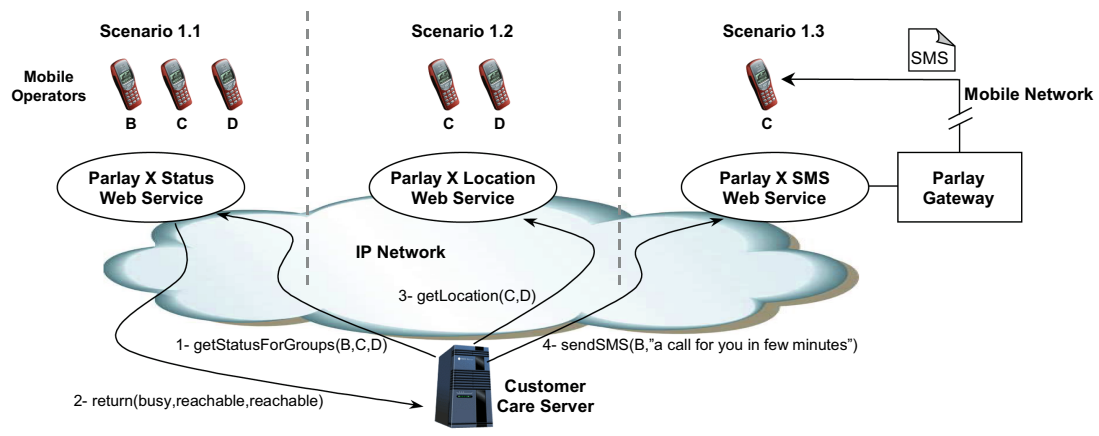

Fig. 5. Parlay terminal status(a), terminal location(b) and SMS(c) Web services usage

In the end of the scenario, the service transparently establishes a call between the user and the operator, using the Third Party Call Web service (see Fig. 6). The choice of calling the nearest available operator enables the reduction of the connection cost, which is an advantage for the user.

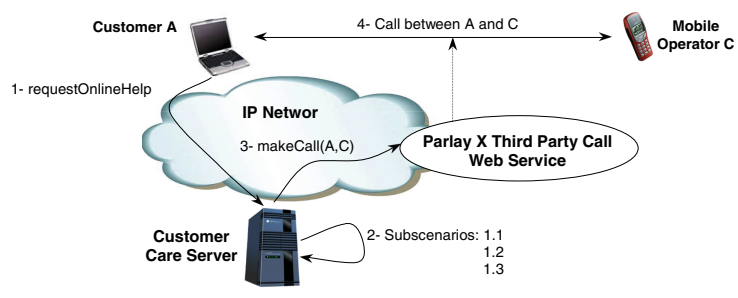

Fig. 6. Parlay third party call Web service usage

\subsection{Multimedia Adaptation Scenario}

This scenario happens when the user requests to access a product tutorial. The service initially uses the Parlay Terminal Capabilities API for obtaining the terminal characteristics, such as the screen dimensions and the audio capabilities (see Fig. 7). With this information, the Customer Care service adapts the SMIL product tutorial accordingly.

When the terminal is a laptop, the produced presentation has the spatial layout displayed in Fig. 7. In this case, no adaptation was performed, since the amount of text and the picture dimensions were all appropriate to fit in the screen dimensions. A different situation occurred when the terminal is a PDA, although the overall presentation structure shown in Fig. 7 is maintained. In this case, a primary adaptation operation is performed over text objects, adjusting the font size to an appropriate value, taking into account the small screen dimensions. 


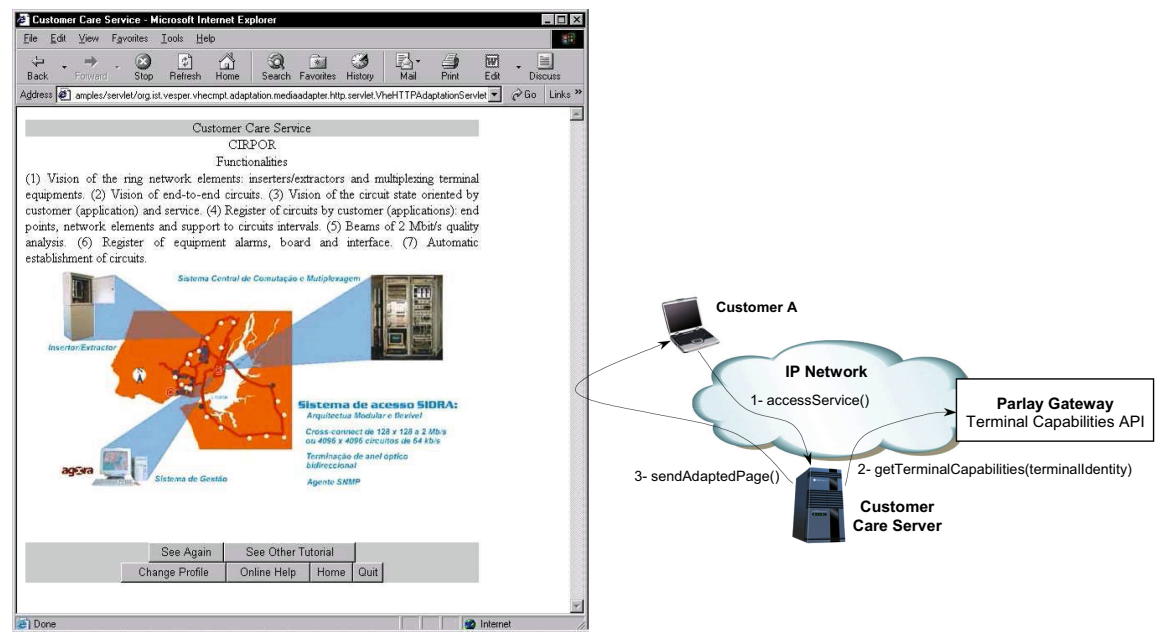

Fig. 7. Parlay terminal capabilities API usage

Then, because the width of some pictures of the tutorial is larger than the screen width, this fact forces the SMIL Media Adapter to adapt them, requesting the use of an image resize media adapter. The resize is performed if the percentage of the reduction does not go beyond a specified threshold, which is defined as a limit to preserve information consistency. If a specific picture needs a resize greater than the mentioned threshold, the SMIL Media Adapter takes the decision to drop it from the tutorial presentation.

As result of analysis, the approach based on the use of dynamic constraints, promoted by the SMIL Media Adapter, performs well with respect to the most common terminals that support multimedia presentations, guaranteeing consistent tutorial presentations even in the face of terminals with less capabilities.

\section{Related Work}

The work on the MModel can be seen as parallel to the definition of the User Interface Markup Language (UIML) [11, which is a declarative language, currently being standardized by OASIS, that derives its syntax from XML and enables the description of device-independent user interfaces. In contrast with UIML, the definition of the MModel did not have the ambition of being a standardized way to define user interfaces but only had the objective of defining a model simple enough to demonstrate the adaptation methodology.

In recent years, some research projects have been dedicated to the development of multimedia presentation systems following the constraint-based approach. Cuypers [12 is a research prototype system for the generation of Web-based multimedia presentations. Although our work was greatly inspired 
by this system, mainly because the system is designed to operate in the context of a client/server architecture and also because it is mostly targeted to the Web environment, the two approaches have differences. The most relevant is the dynamic integration of context information in the user interface generation process, promoted by the adaptation methodology proposed in this paper.

The integration of context information in telecommunications services and the development of context-sensitive applications have grown enormously in the present decade, mainly due to the increase mobility of users and to the research activities on ubiquitous and pervasive computing. The SmartRoutaari 13 is a sound example of a context-sensitive system. SmartRotuaari is operational at the city center of Oulu, in Northern Finland, and comprises of a wireless multiaccess network (WLAN, GPRS and EDGE), a middleware architecture for service provisioning, a Web portal with content provider interface and a collection of functional context-aware mobile multimedia services. The contextual information gathered by the SmartRoutaari system include the time, the location, the weather, the user preferences and the presence status. In contrast with our approach, the SmartRoutaari solution for gathering user context information is proprietary and has no facilities for the seamless expansion with independent third party services. This approach is not in line with the current standardization activities on telecommunications service provision, namely the 3GPP IMS standardization effort, which promotes the use of open APIs, such as Parlay, for opening operator networks to trusted third parties.

\section{Conclusions}

The work presented in this paper is a proposal to solve the problem of the dynamic adaptation of multimedia services. We presented a generic adaptation methodology suitable for the adaptation of telecommunications services. The methodology follows the single source, multiple deliveries approach, using a conceptual model (the MModel) for the user interface specification. The MModel enables a device independent specification of multimedia user interfaces, promoting a clear separation between service structure and service presentation. This model should be seen as a powerful way for the rapid construction of context independent representations of multimedia user interfaces, which should then be materialized into the most suitable formats.

We presented the most important implementation aspects of a media adapter prototype (the SMIL Media Adapter) specifically designed to enable the introduction of multimedia contents and to support time-based features in telecommunications services targeted to be used in the Web context. The SMIL Media Adapter relies on the use of open middleware, such as the Parlay APIs and Web services, to detect user contextual changes in mobile environments, which include the physical properties of the user terminal, the network state and the user location. These changes are then used to generate a set of constraints that the user interface should satisfy so that it may be properly displayed in the current conditions. 


\section{References}

1. Camarillo, G., García-Martín, M.A.: The 3G IP Multimedia Subsystem: Merging the Internet and the Cellular Worlds. John Wiley \& Sons, Chichester (2006)

2. Oliveira, J., Roque, R., Carrapatoso, E., Portschy, H., Hoványi, D., Berenyi, I.: Mobile Multimedia in VESPER Virtual Home Environment. In: Proceedings of the IEEE International Conference on Multimedia and Expo, Lausanne, Switzerland (August 26-29, 2002)

3. Oliveira, J., Roque, R., Dinis, M., Carrapatoso, E.: Provision of Mobile Multimedia over UMTS Middleware Platforms. In: Proceedings of the IST Mobile and Wireless Communications Summit, Aveiro, Portugal (June 15-18, 2003)

4. W3C: Synchronized Multimedia Integration Language (SMIL 2.1) Specification. Recommendation REC-SMIL2-20051213, Synchronized Multimedia Working Group (December 2005)

5. Parlay Group: Parlay Specifications (2003), http://www . parlay.org/en/specifications

6. W3C: Composite Capability/Preference Profiles (CC/PP): Structure and Vocabularies. Recommendation REC-CCPP-struct-vocab-20040115, Device Independence Working Group (January 2004)

7. Kay, M.: XSL Transformations (XSLT) - Version 2.0. Proposed Recommendation PR-xslt20-20061121, W3C (November 2006)

8. Bulterman, D.C.A.: SMIL 2.0, Part 1: Overview, Concepts and Structure. IEEE Multimedia 8(4), 82-88 (2001)

9. W3C: XForms 1.0. Recommendation REC-xforms-20060314, XForms Working Group (March 2006)

10. Apt, K., Wallace, M.: Constraint Logic Programming using ECLiPSe. Cambridge University Press, Cambridge (2006)

11. Phanouriou, C.: UIML: A Device-Independent User Interface Markup Language. $\mathrm{PhD}$ thesis, Virginia Polytechnic Institute and State University (September 26, 2000)

12. van Ossenbruggen, J., Geurts, J., Cornelissen, F., Hardman, L., Rutledge, L.: Towards Second and Third Generation Web-Based Multimedia. In: Proceedings of the $10^{\text {th }}$ International World Wide Web Conference, Hong Kong, pp. 479-488 (2001)

13. Ojala, T., Korhonen, J., Aittola, M., Ollila, M., Koivumäki, T., Tähtinen, J., Karjaluoto, H.: SmartRotuaari - Context-Aware Mobile Multimedia Services. In: Proceedings of the $2^{\text {nd }}$ International Conference on Mobile and Ubiquitous Multimedia, Norrköping, Sweden (December 10-12, 2003) 